\title{
Increased number of activated microglia in rat spinal cord during early stage of diabetic induction
}

\author{
P. Lanlua, A. Prommahom, S. Sricharoenvej \\ Department of Anatomy, Faculty of Medicine Siriraj Hospital, Mahidol University, Bangkok, Thailand
}

[Received: 26 August 2019; Accepted: 2 December 2019]

Background: Diabetic neuropathy is a common chronic complication in diabetes mellitus. Such neuropathy associates with chronic inflammation and immune system activation. Microglia, a type of neuroglia, are involved in the immune system and are found in grey and white matter of the central nervous system, such as the brain and spinal cord. The spinal cord connects the peripheral nervous system and the higher brain centre. Hyperglycaemia during diabetes mellitus has been found to activate and increase number of microglia in the dorsal grey horn or column of the lumbar segments in spinal cord, which release several cytokines in the development of hypersensitivity in diabetic neuropathic pain.

Materials and methods: Therefore, in this study, anatomical alterations of rat spinal microglia in all areas (dorsal, intermediate and ventral columns of grey matter and dorsal, lateral and ventral funiculi of white matter) in cervical enlargement, thoracic level and lumbosacral enlargement were observed in early stage of diabetic conditions by using light and transmission electron microscopies.

Results: The numbers of microglia in all parts of grey and white matter of all spinal levels significantly increased in the diabetic group. The structures and ultrastructures of microglia in grey and white matter at cervical enlargement, thoracic level and lumbosacral enlargement similarly changed in diabetes. In diabetic rats, microglia became hypertrophied with a pale nucleus. Moreover, short fragments of rough endoplasmic reticulum, elevated numbers of lysosomes and numerous actin filaments in the cytoplasm were examined. Microglial phagocytosis of myelin and axonal debris were also observed. In this investigation, the morphology of spinal microglia during short-term diabetes became activated during hyperglycaemia. Conclusions: It is suggested that these changes may be involved in the development of diabetic neuropathic pain in the spinal cord. (Folia Morphol 2020; 79, 4: 662-671)

Key words: diabetes mellitus, microglia, spinal cord, rat

\section{INTRODUCTION}

Diabetes mellitus (DM) is a chronic metabolic disorder of global concern [11]. Chronic inflammation and activation of the immune system are involved in the pathogenesis of diabetes and associated with diabetic complications, particularly diabetic neuro- pathy [10]. The current hypothesis suggests that neuro-immune interactions actively contribute to pain in diabetes of both peripheral and central nervous systems (CNS) [30]. The cells, which relate with the immune system in the CNS, are microglia, which are the macrophage-like cells of both white and grey

Address for correspondence: Dr. S. Sricharoenvej, Department of Anatomy, Faculty of Medicine Siriraj Hospital, Mahidol University, 2 Wanglang Road, Bangkoknoi, Bangkok 10700, Thailand, tel: +662-419-8592, fax: +662-419-8523, e-mail: sirinush.sri@mahidol.ac.th 
matter [17]. The microglia can be classified into two different forms with varying functional roles: resting in the normal condition and active microglia in neural damage, ischaemia and infection [4]. Furthermore, the high concentration of glucose during short-term diabetic rats can activate and increase in number of microglia, which is observed in dorsal horn or column of grey matter in spinal lumbar segments [24]. The activation of microglia is implicated in stimulation of intracellular signalling molecules and release of pro-inflammatory cytokines, which are involved in pain hypersensitivity in diabetic neuropathy $[3,5,14$, 23]. Most previous studies have focused on biochemical alterations of microglia. However, little is known regarding the beginning of phenotypic changes of microglia in all areas and levels of spinal cord, which receive and modulate the sensory input from peripheral nerves to the higher centre and also output to peripheral organs [7]. Thus, this study aimed to investigate the numbers and activated morphological changes of early diabetic spinal microglia in all parts at the cervical enlargement, thoracic level and lumbosacral enlargement by using light (LM) and transmission electron microscopies (TEM), compared to untreated control rats.

\section{MATERIALS AND METHODS}

\section{Experimental animals, LM and TEM}

Fifteen healthy male Sprague-Dawley rats, 5-8 weeks of age, initially weighting 200-270 g, were received from the National Laboratory Animal Centre, Mahidol University, Thailand. The animal protocol was followed by Mahidol University Council's Guidelines for Care and Use of Laboratory Animals, Thailand. After arriving, the rats were acclimatised for a week. Then, all rats were fasted at least $6 \mathrm{~h}$ to measure blood glucose of tail vein and urine glucose. Both levels were in the normal ranges. Then, the animals were randomly divided into two groups: a type 1 -induced DM $(n=9)$ with an intraperitoneal injection of $60 \mathrm{mg} / \mathrm{kg}$ body weight of streptozotocin (STZ) in citrate buffer and a control $(n=6)$, injected with the buffer [27]. After fasting for 10-12 $h$, urine glucose levels and body weights of each animal were measured daily for 4 weeks. The whole blood glucose levels were detected at $48 \mathrm{~h}, 72 \mathrm{~h}$ after the STZ or the buffer injection and before sacrifice. Each animal was sacrificed by an overdose of halothane inhalation at 4 weeks after induction.

In the $\mathrm{LM}$, after $500 \mathrm{~mL}$ of $0.9 \%$ sodium chloride was injected through the ascending aorta, $300 \mathrm{~mL}$ of the Bouin's solution was followed. Next, 6 spinal cords (control: $n=3, D M: n=3$ ) were dissected into cervical enlargement, thoracic level and lumbosacral enlargement, cut at 6-7 $\mu \mathrm{m}$ thick and processed for conventional histological investigation with haematoxylin $(\mathrm{H})$ and eosin $(\mathrm{E})$ staining.

In the TEM, 0.1 M phosphate buffered saline (PBS) was perfused into ascending aorta and then injected with $2.5 \%$ glutaraldehyde in $0.1 \mathrm{M}$ PBS, orderly. Nine rat spinal cords (control: $n=3, D M: n=6$ ) were dissected and separated into cervical enlargement, thoracic level and lumbosacral enlargement. Next, each level of spinal cord was cut into $1 \mathrm{~mm}$ cube, according to dorsal, lateral and ventral funiculi of white matter and dorsal, intermediate and ventral columns of grey matter. Then, each area of each spinal level was processed for the conventional TEM [19, 22].

\section{Microglia counting}

In all areas of grey and white matter in all spinal levels (dorsal, intermediate and ventral columns of grey matter, dorsal, lateral and ventral funiculi of white matter in cervical enlargement, thoracic level and lumbosacral enlargement in both control and diabetic groups), the numbers of microglia were counted under the LM with 400 magnification. The amounts of microglia per area $\left(200 \times 300 \mu \mathrm{m}^{2}\right)$ in each area, spinal level and group were totally counted in 30 serial sections with 3 section interval in each counted section; therefore the same microglia was not repeatedly summed. Amounts of microglia in the same area of each level and group were calculated as an average and a standard deviation (SD).

\section{Statistical analysis}

The quantitative analyses in body weights and numbers of microglia in each area, level and group were expressed as means \pm SD. The Mann-Whitney $U$ test was used to compare body weights of control and diabetic groups. Comparisons in amounts of microglia between control and diabetic groups in each area and level were performed using unpaired t-test (SPSS 19.0 software). Differences were considered significant at $p$-values $<0.05$.

\section{RESULTS}

At $48 \mathrm{~h}$ and $72 \mathrm{~h}$ after the STZ induction and before sacrifice, the average whole blood glucose level of diabetic rats was higher than $300 \mathrm{mg} / \mathrm{dL}$, and the 
Table 1. Comparisons of parameters between early stage of diabetic rats, compared to age-matched control rats (mean \pm standard deviation)

\begin{tabular}{lcc}
\hline Parameters & Control $(\mathbf{n}=\mathbf{6})$ & Diabetes $(\mathbf{n}=\mathbf{9})$ \\
\hline $\begin{array}{l}\text { Whole blood glucose levels } \\
{[\mathrm{mg} / \mathrm{dL}] \text { : }}\end{array}$ & & \\
$\quad \begin{array}{l}\text { Before experiment } \\
\text { Before sacrifice }\end{array}$ & $86.50 \pm 3.37$ & $85.00 \pm 1.00$ \\
$\begin{array}{l}\text { Urine glucose levels [mg/dL]: } \\
\quad \text { Before experiment }\end{array}$ & 0 & 0 \\
$\quad$ Before sacrifice & 0 & $>500^{\mathrm{b}}$ \\
Body weights [g]: & & \\
$\quad$ Before experiment & & \\
$\quad$ Before sacrifice & $253.67 \pm 66.18$ & $252.38 \pm 38.51$ \\
\hline
\end{tabular}

alf the average whole blood glucose level was more than $300 \mathrm{mg} / \mathrm{dL}$, it was indicated as diabetes in rat; ' lff the average urine glucose level was greater than $500 \mathrm{mg} / \mathrm{dL}$, it was a characteristic of diabetic rat; ${ }^{*} p$-value $<0.05$ compared to the age-matched control rats

mean urine glucose level of diabetic rats was more than $500 \mathrm{mg} / \mathrm{dL}$, which indicated that these rats became diabetes. Moreover, the diabetic rats showed other symptoms of DM, including polyphagia, polydipsia, and polyuria. Moreover, there was a significant decrease in body weight of early stage in diabetes, compared to the control (Table 1).

\section{Altered histological appearances and numbers of microglia in early diabetes}

At low magnification, the cross sections of spinal cords in cervical enlargement, thoracic level and lumbosacral enlargement consisted of inner butterfly shaped grey and outer surrounding white matter. The grey matter was divided into dorsal, intermediate and ventral columns. The white matter was classified into dorsal, lateral and ventral funiculi. In the diabetes, the spinal cords were slightly paler than those in the control group (Fig. 1). Microglial appearances of each group (control and diabetes) in all white and grey matter of cervical enlargement, thoracic level and lumbosacral enlargement in spinal cord were similar in both histological and ultrastructural examinations. Under LM observation, microglia in grey matter dispersed among neurons, other neuroglial cells and blood vessels (Fig. 2), whereas they were situated around nerve fibres and other neuroglia in white matter (Fig. 3). To identify glial cells (microglia, astrocytes and oligodendrocytes) of both grey and white matter with the H\&E staining, a microglia had a smaller and more elongated nucleus with dense basophilic staining, whereas an astrocyte contained a large oval nucleus with pale staining. Moreover, an oligodendrocyte consisted of a smaller and dense round nucleus, compared to the astrocyte (Figs. 2, 3). In the diabetes, microglia became slight hypertrophied with a pale-stained nucleus in all areas (dorsal, intermediate and ventral columns of grey matter and dorsal, lateral and ventral funiculi of white matter) in all levels (cervical enlargement, thoracic level and lumbosacral enlargement) of spinal cords (Figs. 2, 3). Interestingly, the disorganised pattern of nerve fibres in white matter was detected in diabetes (Fig. 3). Moreover, the numbers of diabetic microglial cells significantly increased compared to the age-matched control group in all parts of all spinal levels (Figs. 2-4).

\section{Ultrastructural changes in early stage of diabetic microglia}

Under TEM, the rod-shaped nuclei of control microglial cells were dark with clumped chromatin beneath the nuclear envelope. The contours of cell bodies were irregular and extended thin cytoplasmic processes into the surrounding neuropil (Fig. $5 A, B)$. The cells in the control contained a nucleus and numerous organelles, including long cisternae of rough endoplasmic reticulum ( $r E R$ ) and oval or elongated mitochondria (Fig. 6A-C). In addition, a few fine fibrillar actin filaments arranged in parallel arrays in the cytoplasm were observed (Fig. 6B). In short-term diabetes, the altered microglial cell had an oval shape nucleus with dense thick patches of heterochromatin along the periphery with extension of microglial processes in comparison to the control (Fig. 5C-H). Moreover, a broad cytoplasmic area was found with several changes of cytoplasmic organelles. Short segments of $r E R$ and spherical lysosomes partly filled with hydrolytic enzymes were widely dispersed in the diabetic microglial cytoplasm (Fig. 6D-F). An abundance of actin filaments was also observed near the nucleus of diabetic microglia (Figs. 5D, G; 6E). Furthermore, the degenerated myelin and axonal debris were found in the cytoplasm, which suggested phagocytic activity during diabetes (Fig. 6F).

\section{DISCUSSION}

In early stage of diabetes, increased numbers of microglia were found in this investigation of all parts of spinal grey and white matter (dorsal, intermediate and ventral columns of grey matter and dorsal, lateral and ventral funiculi of white matter) 


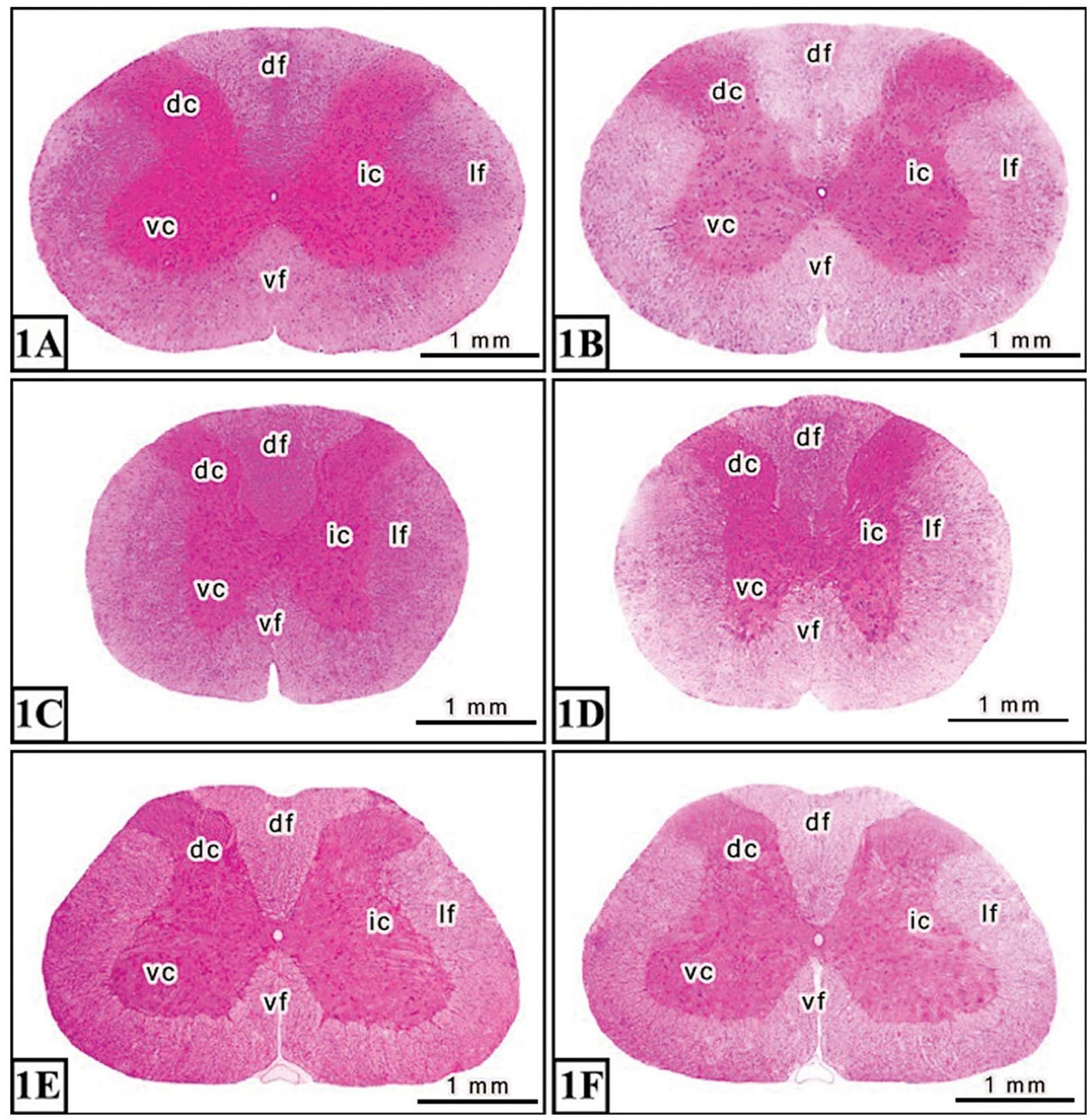

Figure 1. Representative light microscope of cross sections in cervical enlargement (A, B), thoracic level (C, D) and lumbosacral enlargement (E, F) of spinal cord, short-term control (A, C, E) and diabetes (B, D, F). Dorsal (dc), intermediate (ic) and ventral columns (vc) of grey matter, dorsal (df), lateral (If) and ventral funiculi (vf) of white matter.

in cervical enlargement, thoracic level and lumbosacral enlargement, which was similar result as that in dorsal horn or column of spinal lumbar segments in the short-term DM [24]. Hyperglycaemia during DM leads to non-enzymatic glycation to produce advanced glycation end products (AGEs). Then, AGEs activate oxidative stress via activation of nicotinamide adenine dinucleotide phosphate oxidase [28], which produces hydrogen peroxide $\left(\mathrm{H}_{2} \mathrm{O}_{2}\right)$ in the microglia. $\mathrm{H}_{2} \mathrm{O}_{2}$ then stimulates sulfhydryl groups in the tyrosine phosphatase, which causes increased tyrosine phosphorylation and prolongation of mitogenic signalling [9]. Consequently, haematopoietic cell kinase activation occurs to induce phosphatidylinositol-3 kinase (PI3K)/protein kinase B (Akt) and mitogen-activated protein kinases (MAPK)/extracellular signal-regulated kinase pathways [20]. As a result, cyclin D1 and cyclin-dependent kinase (CDK) 4 increase to progress $G_{0}$ to $G_{1}$ phases, whereas raised levels of cyclin $E$ and $C D K_{2}$ cause $G_{1}$ to $S$ phases of 

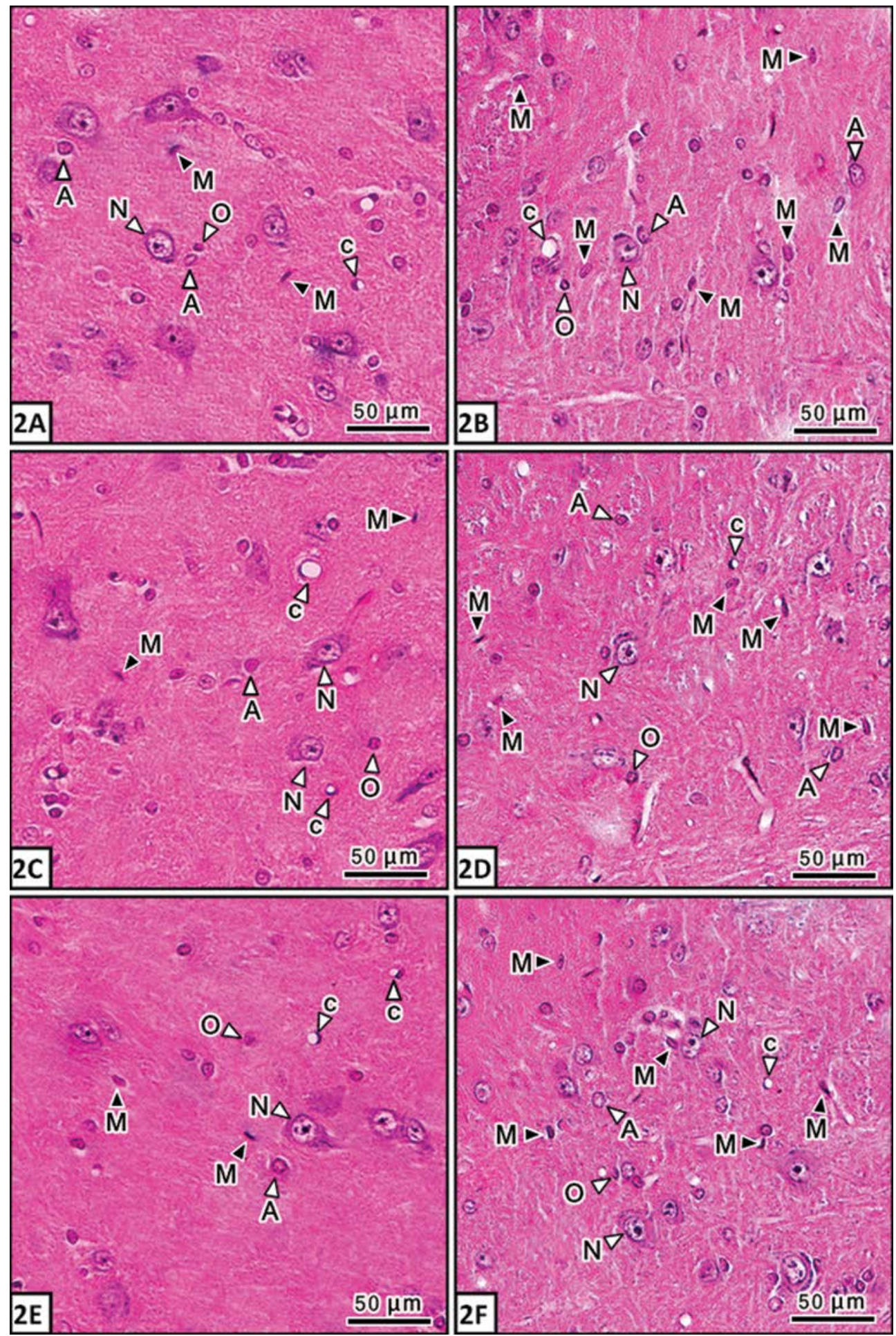

Figure 2. Light microscope of spinal glial cell types in typical grey matter of cervical enlargement (A, B), thoracic level (C, D) and lumbosacral enlargement $(\mathbf{E}, \mathbf{F})$ in control $(\mathbf{A}, \mathbf{C}, \mathbf{E})$ and early stage of $\mathrm{DM}(\mathbf{B}, \mathbf{D}, \mathbf{F})$. M - microglia; $\mathrm{A}$ — astrocyte; $\mathrm{O}$ — oligodendrocyte; $\mathrm{N}$ — neuron; $\mathrm{C}$ - capillary.

the cell cycle. Moreover, decreased CDK inhibitor, p2 $27^{\text {Kip } 1}$, prevents the cell cycle from moving to the $G_{0}$ phase [21]. Finally, proliferation and differentiation of diabetic microglia caused significantly increased numbers in all areas of both grey and white matter in all levels (cervical enlargement, thoracic level and lumbosacral enlargement) of the spinal cords.

In our experiments, microglia in the early stage of diabetes became hypertrophied in all areas of grey and white matter in cervical enlargement, thoracic 

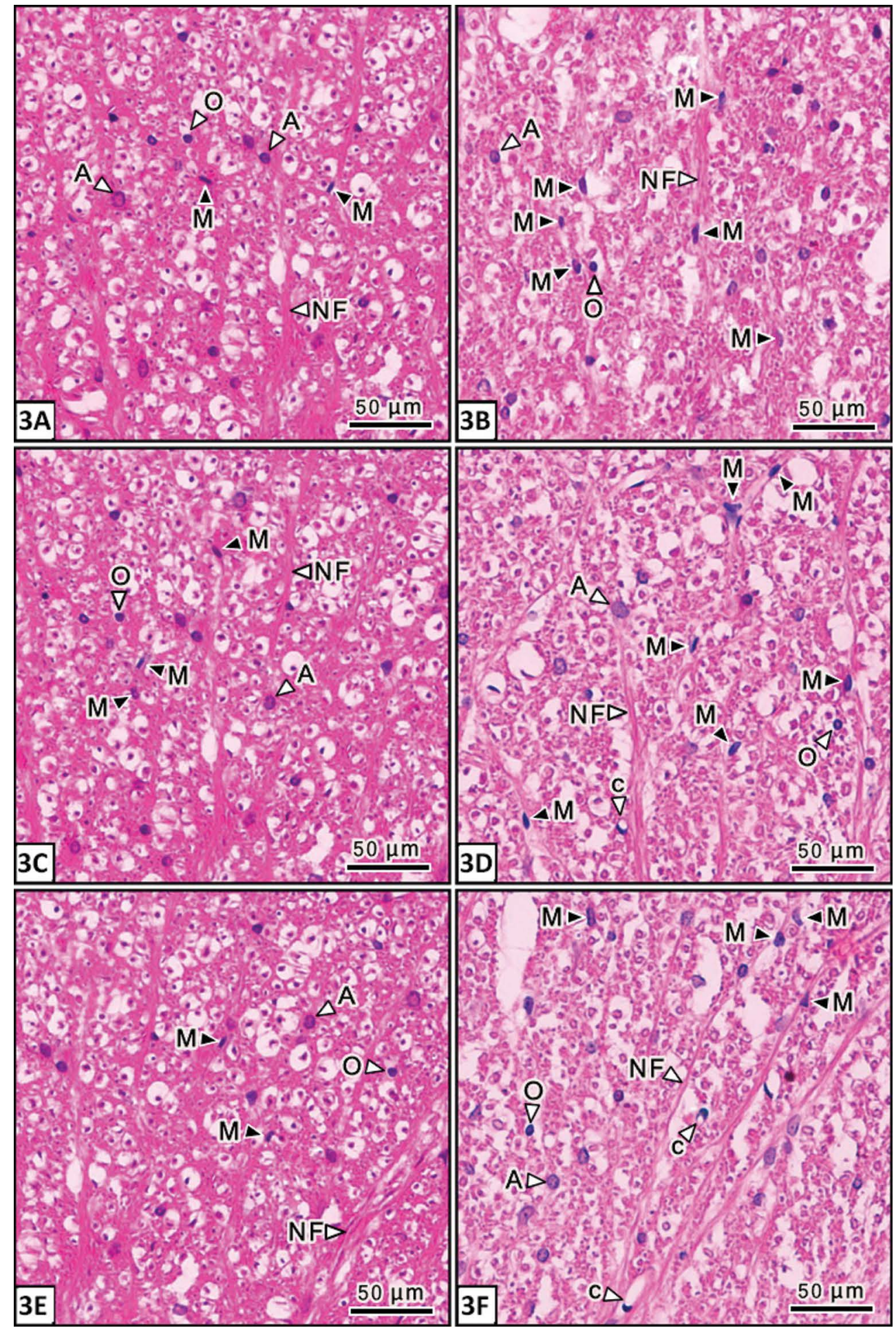

Figure 3. Light microscope of microglia and other cells in white matter of cervical enlargement (A, B), thoracic level (C, D), and lumbosacral enlargement (E, F) in control (A, C, E) and diabetic (B, D, F) groups. M - microglia; A - astrocyte; 0 - oligodendrocyte; NF — nerve fibre; C - capillary.

level and lumbosacral enlargement, which were same appearances as those of the microglia in dorsal horn or column of spinal lumbar segment in short-term diabetes [24]. There were also elevated lysosomes and numerous actin filaments in the diabetic microglia, which are involved in phagocytosis. As described, 
(4A)

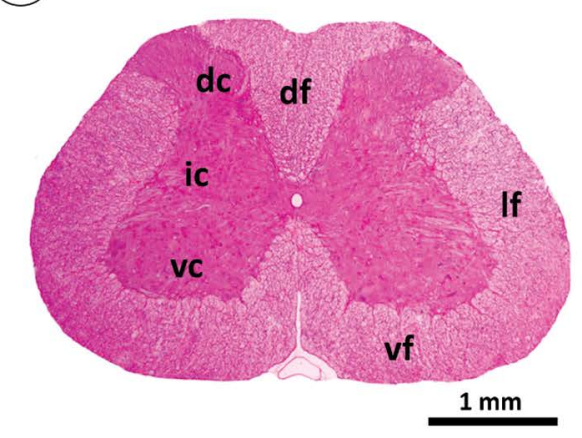

(4C)

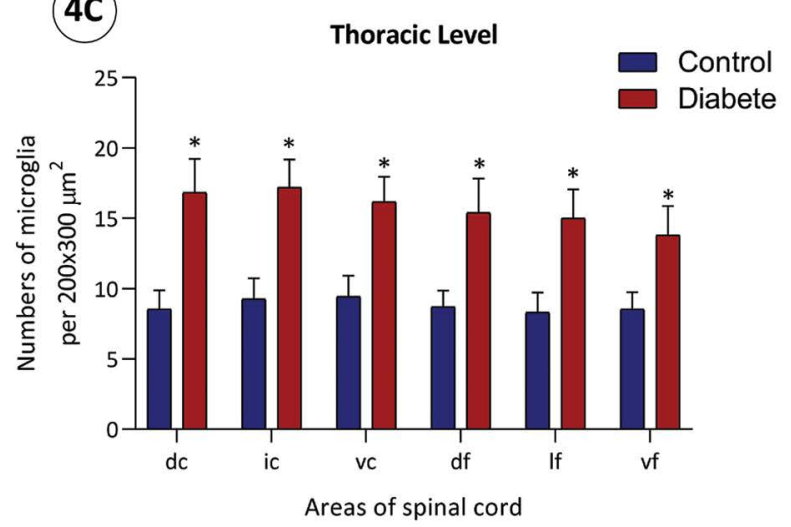

(4B)

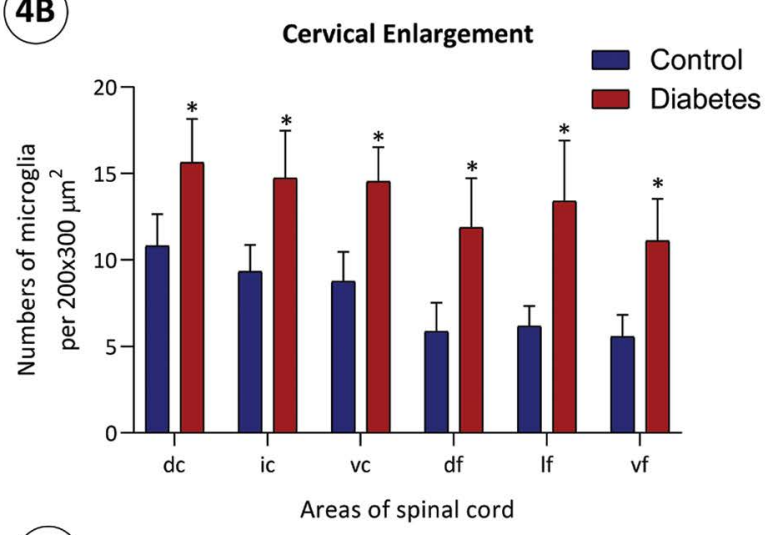

(4D)

Lumbosacral Enlargement

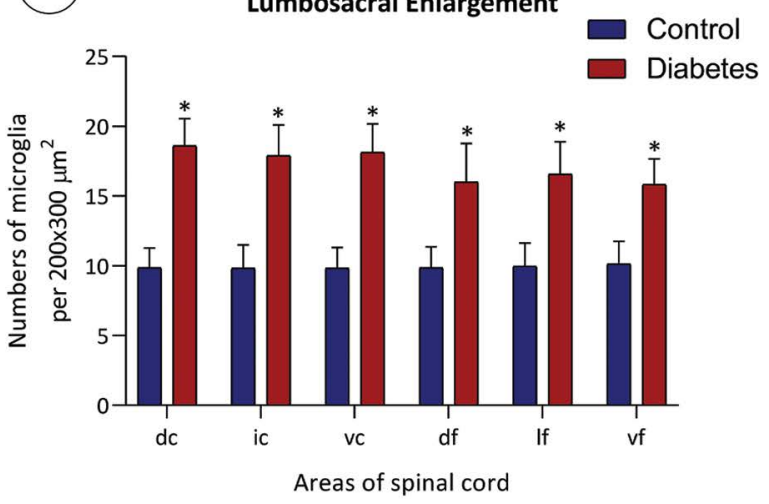

Figure 4. A. A typical cross section of spinal cord; B-D. Comparisons in numbers of microglia per $200 \times 300 \mu \mathrm{m}^{2}$ of cervical enlargement (B), thoracic level (C) and lumbosacral enlargement (D). Dorsal (dc), intermediate (ic) and ventral columns (vc) of grey matter, dorsal (df), lateral (If) and ventral funiculi (vf) of white matter; ${ }^{*} \mathrm{p}$-value $<0.05$ compared to the age-matched control rats.

increased $\mathrm{H}_{2} \mathrm{O}_{2}$ in the microglia activates PI3K/Akt and p38 MAPK pathways [25]. Next, mammalian target of rapamycin is stimulated to increase global protein synthesis and ribosomal gene transcription [1], which directly phosphorylates transcription factor EB to bind and encode the V-ATPase lysosomal gene. Then, $r E R$ becomes hyperfunction to produce lysosomal membrane and hydrolytic enzymes [18]. Therefore, the number of lysosomes in the diabetic microglia increased. In addition, high glucose levels lead to damaged nervous tissues and endothelium [13], which release vascular endothelial growth factor (VEGF) $[26,29]$ and monocyte chemoattractant protein-1 (MCP-1) to microglia. The VEGF activates myocardin-related transcription and serum response factors in that order. Increased G-actin is found [15]. Furthermore, MCP-1 binds to G-couple receptor on the plasma membrane of microglia to stimulate guanosine triphosphate-binding protein [12]. Then, profilin, an actin-binding protein, un- dergoes phosphorylation, while cofilin, involved in depolymerisation of actin filaments, becomes down-regulated in the microglia. Increased numbers of G-actin and profilin, but decreased cofilin, cause elevated production of actin filaments [16]. Abundant actin filaments were found in the microglia of diabetes. During hyperglycaemia, neurons in the spinal cord are damaged and become apoptotic cells, releasing uridine $5^{\prime}$-diphosphate. This substance binds to its receptor on the microglia to produce phospholipase $\mathrm{C}$ in the PI3K pathway. The inositol 1, 4, 5-trisphosphate (InsP3) is relased to cause calcium $\left(\mathrm{Ca}^{2+}\right)$ efflux through InsP3 receptor on the ER [8]. Intracellular $\mathrm{Ca}^{2+}$ induces calcium release-activated channels on the plasma membrane of microglia. Then, $\mathrm{Ca}^{2+}$ flows into the cell [16]. The high level of $\mathrm{Ca}^{2+}$ regulates remodelling of increased actin filaments to form a phagocytic cup around apoptotic debris. Next, the actin filaments surrounding the phagosome dissociate, and lysosomes bind to the phagosome 

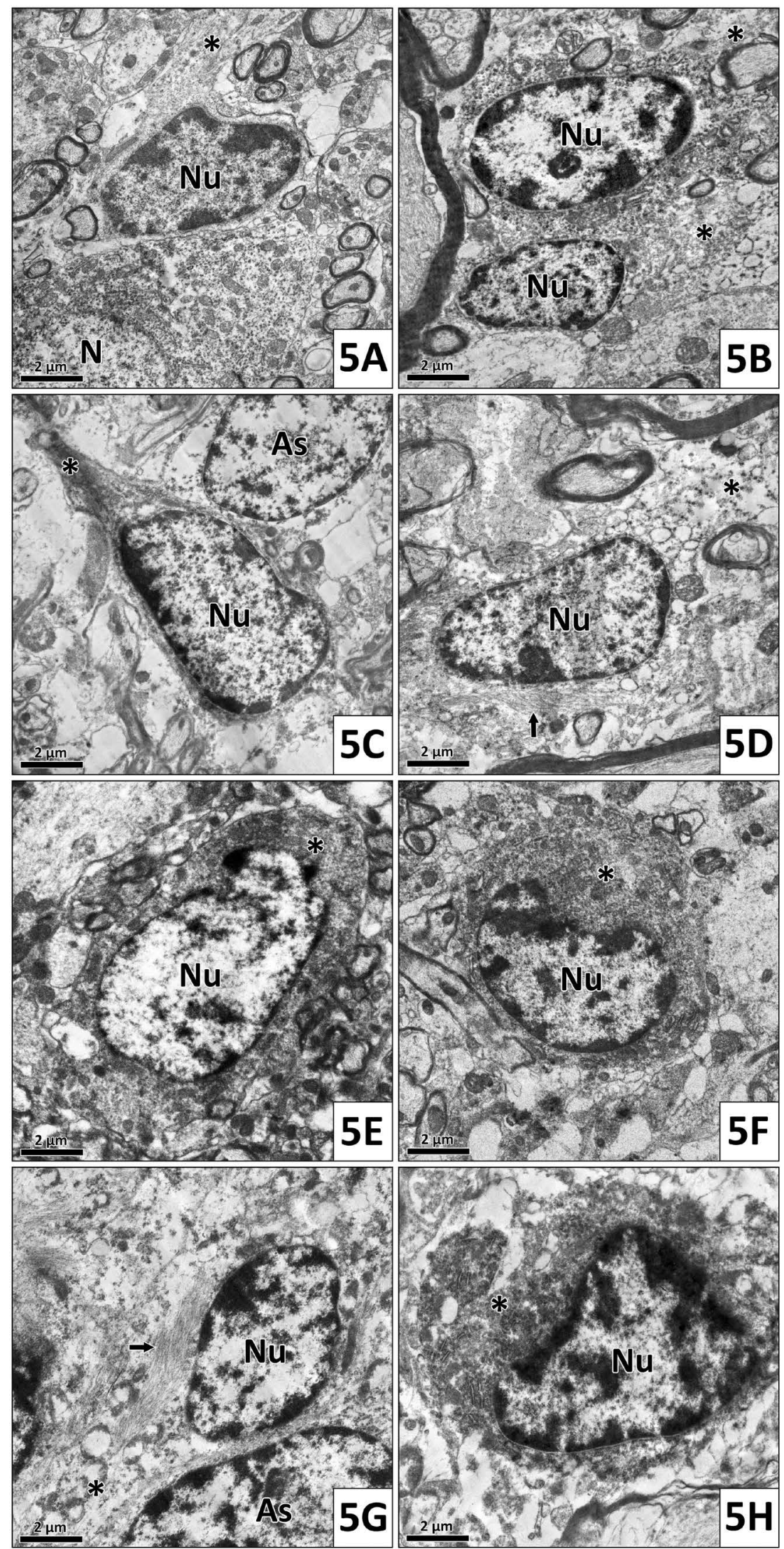

Figure 5. Transmission electron microscope of microglia in representative grey (A) and white matter (B) of control group; grey (C, E, G) and white matter $(\mathbf{D}, \mathbf{F}, \mathbf{H})$ of short-term diabetes mellitus; cervical enlargement $(\mathbf{C}, \mathbf{D})$, thoracic level $(\mathbf{E}, \mathbf{F})$ and lumbosacral enlargement $(\mathbf{G}, \mathbf{H})$; $\mathrm{Nu}$ — nucleus of microglia; black asterisks — microglial process; black arrows — actin filaments; $\mathrm{N}$ — neuron; As — astrocyte. 

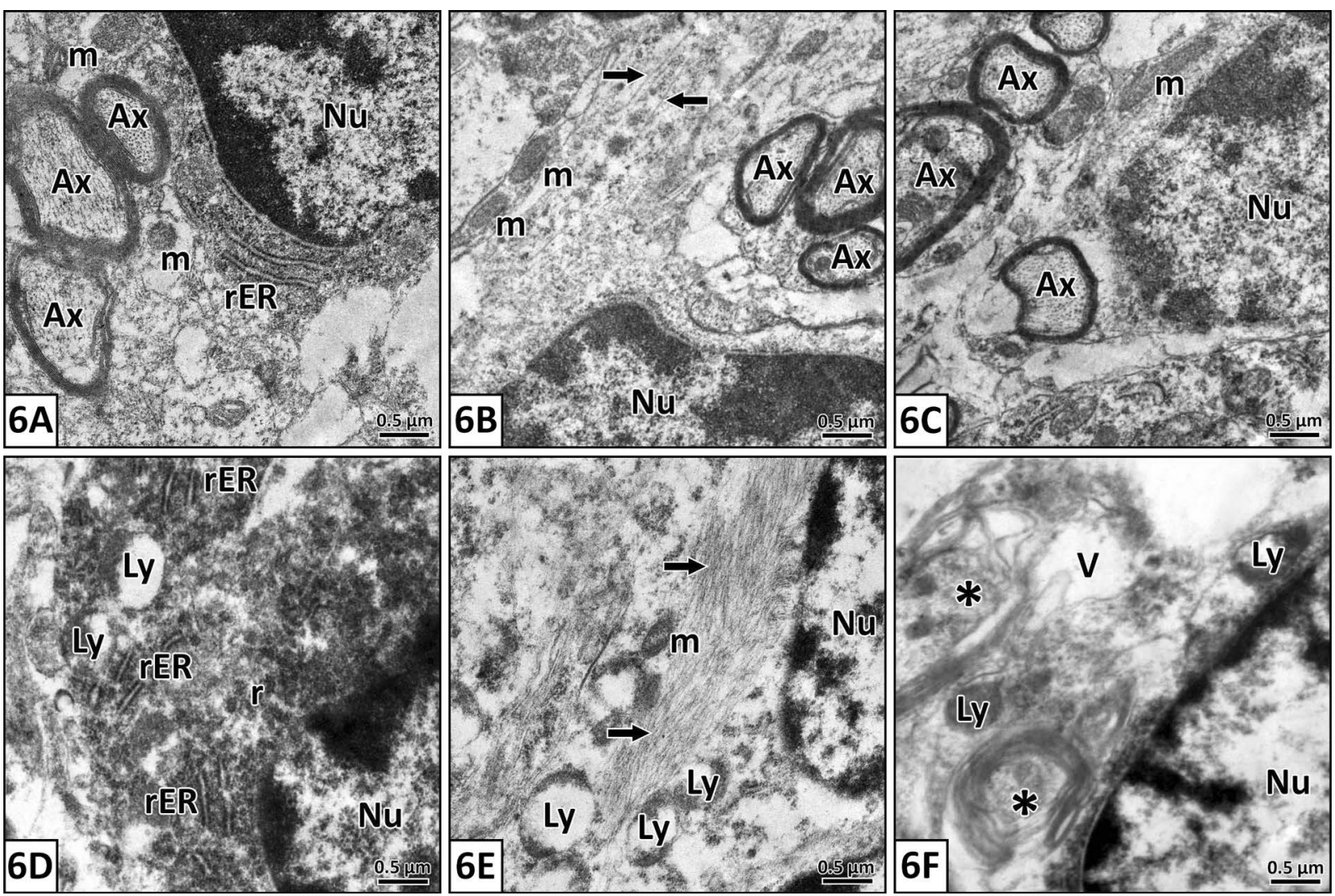

Figure 6. Ultrastructural images of microglial cells in the control (A-C) and streptozotocin diabetic (D-F) groups. Nu — nucleus of microglia; $\mathrm{m}$ - mitochondria, $\mathrm{rER}$ - flatten cisternae rough endoplasmic reticulum; black arrows — actin filaments; $\mathrm{r}$ — ribosome; Ly — lysosome; black asterisks - axonal debris; $\mathrm{V}$ - a vacuole; $\mathrm{Ax}$ — axon.

to digest the debris in the $\mathrm{Ca}^{2+}$ dependency $[2,6]$. Then, phagocytosis of the damaged cells is proceeded by microglia, as seen in the axonal debris in the cytoplasm.

\section{CONCLUSIONS}

During early stage of DM, microglia in all areas of both grey and white matter in cervical enlargement, thoracic level and lumbosacral enlargement became hypertrophied with a significantly increased number. Additionally, the morphological changes in the period of DM contained an oval nucleus with heterochromatin with altered cytoplasmic organelles as in phagocytosis.

\section{Acknowledgements}

This study was supported by the Siriraj Research Fund, Chalermphrakiat Grant, and Siriraj Graduate Scholarship 2014, Faculty of Medicine Siriraj Hospital, Mahidol University, Thailand.

\section{REFERENCES}

1. Ballou LM, Lin RZ. Rapamycin and mTOR kinase inhibitors. J Chem Biol. 2008; 1(1-4): 27-36, doi: 10.1007/s12154008-0003-5, indexed in Pubmed: 19568796.

2. Berridge MJ. Inositol triphosphate and calcium signaling. In: Lennarz WJ, Lane MD, editors. Encyclopedia of Biological Chemistry. 2nd ed. Academic Press, Amsterdam 2013: 583-589.

3. Cheng KI, Wang HC, Chuang YT, et al. Persistent mechanical allodynia positively correlates with an increase in activated microglia and increased P-p38 mitogen-activated protein kinase activation in streptozotocin-induced diabetic rats. Eur J Pain. 2014; 18(2): 162-173, doi: 10.1002/j.15322149.2013.00356.x, indexed in Pubmed: 23868758.

4. Correale J. The role of microglial activation in disease progression. Mult Scler. 2014; 20(10): 1288-1295, doi: 10.1177/1352458514533230, indexed in Pubmed: 24812046

5. Daulhac L, Mallet C, Courteix C, et al. Diabetes-induced mechanical hyperalgesia involves spinal mitogen-activated protein kinase activation in neurons and microglia via $\mathrm{N}$-methyl-D-aspartate-dependent mechanisms. Mol Pharmacol. 2006; 70(4): 1246-1254, doi: 10.1124/ mol.106.025478, indexed in Pubmed: 16868181. 
6. Fu R, Shen $\mathrm{Q}, \mathrm{Xu} \mathrm{P}$, et al. Phagocytosis of microglia in the central nervous system diseases. Mol Neurobiol. 2014; 49(3): 1422-1434, doi: 10.1007/s12035-013-8620-6, indexed in Pubmed: 24395130.

7. Kawano T. A current overview of diabetic neuropathy mechanisms, symptoms, diagnosis, and treatment. Periph Neurop. 2014, doi: 10.5772/58308.

8. Koizumi S, Shigemoto-Mogami Y, Nasu-Tada K, et al. UDP acting at P2Y6 receptors is a mediator of microglial phagocytosis. Nature. 2007; 446(7139): 1091-1095, doi: 10.1038/nature05704, indexed in Pubmed: 17410128.

9. Mander P, Jekabsone A, Brown GK, et al. Microglia proliferation is regulated by hydrogen peroxide from NADPH oxidase. J Immunol. 2006; 176(2): 1046-1052, doi: 10.4049/ jimmunol.176.2.1046, indexed in Pubmed: 16393992.

10. Navale AM, Paranjape AN. Role of inflammation in development of diabetic complications and commonly used inflammatory markers with respect to diabetic complications. Int J Pharm Pharm Sci. 2013; 5(suppl 2): 1-5.

11. Navarro JF, Mora C. Role of inflammation in diabetic complications. Nephrol Dial Transplant. 2005; 20(12): 2601-2604, doi: 10.1093/ndt/gfi155, indexed in Pubmed: 16188894.

12. Olson EN, Nordheim A. Linking actin dynamics and gene transcription to drive cellular motile functions. Nat Rev Mol Cell Biol. 2010; 11(5): 353-365, doi: 10.1038/nrm2890, indexed in Pubmed: 20414257.

13. Peña-Llopis S, Brugarolas J. TFEB, a novel mTORC1 effector implicated in lysosome biogenesis, endocytosis and autophagy. Cell Cycle. 2011; 10(23): 3987-3988, doi: 10.4161/ cc.10.23.18251, indexed in Pubmed: 22101272.

14. Quan Yi, Du J, Wang X. High glucose stimulates GRO secretion from rat microglia via ROS, PKC, and NF-kappaB pathways. J Neurosci Res. 2007; 85(14): 3150-3159, doi: 10.1002/jnr.21421, indexed in Pubmed: 17639599.

15. Quan $\mathrm{Yi}$, Jiang $\mathrm{Ct}$, Xue $\mathrm{B}$, et al. High glucose stimulates $\mathrm{TNF} \alpha$ and MCP-1 expression in rat microglia via ROS and NF- $\kappa$ B pathways. Acta Pharmacol Sin. 2011; 32(2): 188-193, doi: 10.1038/aps.2010.174, indexed in Pubmed: 21293471.

16. Rajakylä EK, Vartiainen MK. Rho, nuclear actin, and actin-binding proteins in the regulation of transcription and gene expression. Small GTPases. 2014; 5: e27539, doi: 10.4161/sgtp.27539, indexed in Pubmed: 24603113.

17. Rock RB, Gekker G, Hu S, et al. Role of microglia in central nervous system infections. Clin Microbiol Rev. 2004; 17(4): 942-64, table of contents, doi: 10.1128/CMR.17.4.942964.2004, indexed in Pubmed: 15489356.

18. Settembre C, Zoncu R, Medina DL, et al. A lysosome-to-nucleus signalling mechanism senses and regulates the lysosome via mTOR and TFEB. EMBO J. 2012; 31(5): 1095-1108, doi: 10.1038/emboj.2012.32, indexed in Pubmed: 22343943.
19. Sricharoenvej S, Chookliang A, Lanlua $P$, et al. Effects of streptozotocin diabetes on the ultrastructure of astrocytes in the cervical enlargement of rat spinal cord. Scien Res Essays. 2015 ; 10(22): 640-648, doi: 10.5897/sre2015.6330.

20. Suh HS, Kim MO, Lee SC. Inhibition of granulocyte-macrophage colony-stimulating factor signaling and microglial proliferation by anti-CD45RO: role of Hck tyrosine kinase and phosphatidylinositol 3-kinase/Akt. J Immunol. 2005; 174(5): 2712-2719, doi: 10.4049/jimmunol.174.5.2712, indexed in Pubmed: 15728479.

21. Taylor L, Bar-Sagi D. The role of Rac and Rho in cell cycle progression. 2nd edition. Academic Press, Amsterdam. Handbook of Cell Signaling. 2010: 1781-1784, doi: 10.1016/b978-0-12-374145-5.00217-5.

22. Techarang T, Lanlua $P$, Niyomchan A, et al. Epidermal modification in skin of streptozotocin-induced diabetic rats. Walailak J Sci Technol. 2017; 14(8): 671-676.

23. Toth CC, Jedrzejewski NM, Ellis CL, et al. Cannabinoid-mediated modulation of neuropathic pain and microglial accumulation in a model of murine type I diabetic peripheral neuropathic pain. Mol Pain. 2010; 6: 16, doi: 10.1186/1744-8069-6-16, indexed in Pubmed: 20236533.

24. Tsuda $M$, Ueno $H$, Kataoka A, et al. Activation of dorsal horn microglia contributes to diabetes-induced tactile allodynia via extracellular signal-regulated protein kinase signaling. Glia. 2008; 56(4): 378-386, doi: 10.1002/ glia.20623, indexed in Pubmed: 18186080.

25. Wang D, Couture R, Hong Y. Activated microglia in the spinal cord underlies diabetic neuropathic pain. Eur J Pharmacol. 2014; 728: 59-66, doi: 10.1016/j.ejphar.2014.01.057, indexed in Pubmed: 24508519.

26. Willard AL, Herman IM. Vascular complications and diabetes: current therapies and future challenges. J Ophthalmol. 2012; 2012: 209538, doi: 10.1155/2012/209538, indexed in Pubmed: 22272370.

27. Wu J, Yan U. Streptozotocin-induced type 1 diabetes in rodents as a model for studying mitochondrial mechanisms of diabetic $\beta$ cell glucotoxicity. Diabetes Metab Syndr Obes. 2015; 8: 181-188, doi: 10.2147/DMSO.S82272, indexed in Pubmed: 25897251.

28. Yagihashi S, Mizukami H, Sugimoto K. Mechanism of diabetic neuropathy: Where are we now and where to go? J Diabetes Investig. 2011; 2(1): 18-32, doi: 10.1111/j.20401124.2010.00070.x, indexed in Pubmed: 24843457.

29. Zochodne D. Mechanisms of diabetic neuron damage: molecular pathways. In: Zochodne DW, Malik RA, editors. Handbook of Clinical Neurology: diabetes and the nervous system. 3rd edition. Elsevier B.V., Amsterdam. 2014: 379-399, doi: 10.1016/b978-0-444-53480-4.00028-x.

30. Zychowska M, Rojewska E, Przewlocka B, et al. Mechanisms and pharmacology of diabetic neuropathy - experimental and clinical studies. Pharmacol Rep. 2013; 65(6): 1601-1610, doi: 10.1016/s1734-1140(13)71521-4, indexed in Pubmed: 24553008. 